\title{
Packing and Covering Triangles in $K_{4}$-free Planar Graphs*
}

\author{
Penny Haxell ${ }^{\dagger} \quad$ Alexandr Kostochka ${ }^{\ddagger}$ and Stéphan Thomassé ${ }^{\S}$
}

April 21, 2011

\begin{abstract}
We show that every $K_{4}$-free planar graph with at most $\nu$ edge-disjoint triangles contains a set of at most $\frac{3}{2} \nu$ edges whose removal makes the graph triangle-free. Moreover, equality is attained only when $G$ is the edge-disjoint union of 5-wheels plus possibly some edges that are not in triangles. We also show that the same statement is true if instead of planar graphs we consider the class of graphs in which each edge belongs to at most two triangles. In contrast, it is known that for any $c<2$ there are $K_{4}$-free graphs with at most $\nu$ edge-disjoint triangles that need more than $c \nu$ edges to cover all triangles.
\end{abstract}

\section{Introduction}

The main motivation for this paper is an old conjecture of Tuza about packing and covering of triangles by edges. A triangle packing in a graph $G$ is a set of pairwise edge-disjoint triangles. A triangle edge cover in $G$ is a set of edges meeting all triangles. We denote by $\nu(G)$ the maximum cardinality of a triangle packing in $G$, and by $\tau(G)$ the minimum cardinality of a triangle edge cover for $G$. It is clear that for every graph $G$ we have $\nu(G) \leq \tau(G) \leq 3 \nu(G)$.

In 1984, Tuza [11] proposed the following conjecture.

Conjecture 1 For every graph $G, \tau(G) \leq 2 \nu(G)$.

${ }^{*}$ This research was done at the Institute for Pure and Applied Mathematics at UCLA.

$\dagger$ Department of Combinatorics and Optimization, University of Waterloo, Waterloo, ON N2L 3G1, Canada. E-mail address: pehaxell@math.uwaterloo.ca. This author's research was partially supported by NSERC.

${ }_{\ddagger}^{\ddagger}$ Department of Mathematics, University of Illinois, Urbana, IL 61801 USA and Institute of Mathematics, Novosibirsk 630090, Russia. E-mail address: kostochk@math.uiuc.edu. Research of this author is supported in part by NSF grant DMS-0965587 and by grant 09-01-00244 of the Russian Foundation for Basic Research.

$\S$ Université Montpellier II - LIRMM, 34392 Montpellier Cedex 5, France. E-mail address: Stephan.Thomasse@lirmm.fr 
The complete graphs $K_{4}$ and $K_{5}$ show that this bound is tight. The conjecture is known to be true for certain special classes of graphs, for example $K_{5}$-free chordal graphs and planar graphs (Tuza [12]), more generally, graphs without a subdivision of $K_{3,3}$ (Krivelevich [8]), tripartite graphs (Haxell and Kohayakawa [6]), odd-wheel-free graphs and four-colourable graphs (Aparna Lakshmanan, Bujtás and Tuza [1]). Weighted versions of the problem were studied in [2]. The only general bound known [5] shows that $\tau(G) \leq \frac{66}{23} \nu(G)$ for every graph $G$.

Our aim in this paper is to study the planar case more closely. As just mentioned, Tuza [12] proved that the conjecture is true for planar graphs, and it is tight for $K_{4}$. In [3] it was shown that equality holds if and only if $G$ is an edge-disjoint union of copies of $K_{4}$ plus possibly some edges that are not in triangles. Here we consider the next step.

Theorem 1 Let $G$ be a $K_{4}$-free planar graph. Then $\tau(G) \leq \frac{3}{2} \nu(G)$, and equality holds if and only if $G$ is an edge-disjoint union of 5-wheels plus possibly some edges that are not in triangles.

Our main tool will be a similar result for a different class of graphs. A graph $G$ is flat if each edge of $G$ belongs to at most two triangles. Observe that a planar graph is flat if it does not have separating triangles. Flat graphs can be far from planar, but the result we prove for them is the same:

Theorem 2 Let $G$ be a $K_{4}$-free flat graph. Then $\tau(G) \leq \frac{3}{2} \nu(G)$, and equality holds if and only if $G$ is an edge-disjoint union of 5-wheels plus possibly some edges that are not in triangles.

It is worth mentioning that excluding $K_{4}$ does not have a similar effect in the general case of Tuza's Conjecture: for every $\epsilon>0$ there exists a $K_{4}$-free graph $G_{\epsilon}$ satisfying $\tau\left(G_{\epsilon}\right)>(2-\epsilon) \nu\left(G_{\epsilon}\right)$ (see [7]).

Our proof of Theorem 1 makes use of some special properties of the triangle graph $T=T(G)$, defined as follows: the vertices of $T$ are the triangles of $G$, and two vertices are adjacent if the corresponding triangles have an edge in common. These properties are established in Section 2 for flat graphs. Then in Section 3 we prove Theorem 1.

\section{Triangle graphs of flat graphs}

For every flat graph $G$, each edge of the triangle graph $T=T(G)$ naturally corresponds to an edge of $G$. Moreover $T(G)$ is subcubic (i.e. has maximum degree at most three), the parameter $\nu(G)$ is equal to the independence number $\alpha(T)$ of $T$, and $\tau(G)$ is the minimum size $\beta^{\prime}(T)$ of an edge cover of the vertices of $T$. If $T$ has no isolated vertices then by Gallai's Theorem, $\beta^{\prime}(T)+\alpha^{\prime}(T)=|V(T)|$, where $\alpha^{\prime}(T)$ denotes the maximum size of a matching in $T$. Thus to get information on packing and covering triangles in $G$, we will start by studying $\alpha(T)$ and $\alpha^{\prime}(T)$. 
Let $\mathcal{F}$ denote the family of all triangle graphs for flat $K_{4}$-free graphs. Graphs in $\mathcal{F}$ have some interesting properties.

Claim 3 If triangles $B_{1}, B_{2}, B_{3}$ of a flat graph $G$ form a triangle in the triangle graph $T(G)$, then $\left|V\left(B_{1} \cup B_{2} \cup B_{3}\right)\right|=4$.

Proof. Since $B_{1}$ and $B_{2}$ share an edge, $\left|V\left(B_{1} \cup B_{2}\right)\right|=4$. If $B_{3}$ has a vertex not in $B_{1} \cup B_{2}$, then it shares the same edge with both $B_{1}$ and $B_{2}$. But then this edge witnesses that $G$ is not flat.

Claim 4 Each triangle graph $T \in \mathcal{F}$ has no $K_{3}$-subgraphs.

Proof. Suppose that triangles $B_{1}, B_{2}, B_{3}$ induce a $K_{3}$ in $T$. Then by Claim 3 , all these triangles are contained in the same 4-element set, say $M$. But then all the 6 pairs of vertices of $M$ must be edges, and hence $G[M]$ is a $K_{4}$, a contradiction to the definition of $\mathcal{F}$.

Claim 5 Let $G$ be a flat $K_{4}$-free graph. Then any 5 -cycle $\left(B_{1}, \ldots, B_{5}\right)$ in $T(G)$ corresponds to a 5-wheel in $G$.

Proof. Since $B_{2}$ shares two vertices with $B_{1}$ and two vertices with $B_{3}$, there is a vertex $v_{0}$ that belongs to all three triangles. So we have the situation in Fig. 1. Triangle $B_{4}$ must share with $B_{3}$ either $v_{3} v_{4}$ or $v_{0} v_{4}$. If it shares $v_{3} v_{4}$, then since $G$ is $K_{4}$-free, its third vertex, say $v_{5}$, is not $v_{1}$. Then $B_{5}$ needs to share two vertices with each of the two disjoint triangles, $B_{1}$ and $B_{4}$, an impossibility. So, $\left\{v_{0}, v_{4}\right\} \subset B_{4}$. Since $B_{1} B_{4} \notin E(T(G)), v_{5} \neq v_{1}$. Then the only way that a triangle $B_{5}$ shares an edge with $B_{1}$ and an edge with $B_{4}$ is that $v_{0} \in B_{5}$. Since $B_{5} B_{2}, B_{5} B_{3} \notin E(T(G))$, the other two vertices of $B_{5}$ are $v_{1}$ and $v_{5}$.

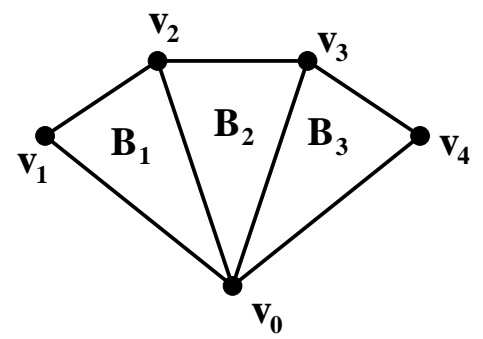

Figure 1:

To study the independence number of graphs in $\mathcal{F}$ we make use of the following theorem of Fajtlowicz [4] and Stanton [10]. 
Theorem 6 Every triangle-free subcubic graph with $n$ vertices has an independent set of size at least $5 n / 14$.

Remark 1. Let $Q$ denote the graph shown in Fig. 2. It is routine to check that $Q$ is the unique triangle-free subcubic graph with 11 vertices and independence number 4 (see Appendix).

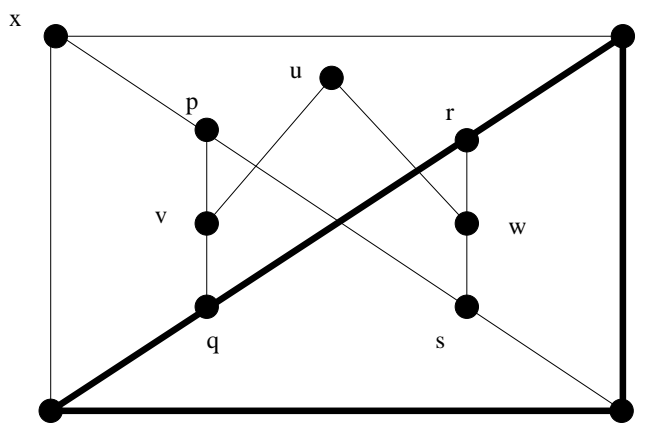

Figure 2: Graph $Q$.

Lemma 7 Let $n$ be odd and $H$ be a triangle-free subcubic graph with $n$ vertices. Then $\alpha(H) \geq(n+1) / 3$. Moreover if equality holds then $H$ is a 5-cycle or a copy of $Q$.

Proof. We note that $5 n / 14>(n+1) / 3$ for $n \geq 15$, so by Theorem 6 we only need to consider odd $n \leq 13$. It is easy to check that the inequality $\lceil 5 n / 14\rceil \geq(n+1) / 3$ also holds for these values (for example, $\left\lceil\frac{5 \cdot 13}{14}\right\rceil=5>\frac{13+1}{3}$ ), and that equality occurs only for $n \in\{5,11\}$. The only 5 -vertex triangle-free graph with independence number 2 is $C_{5}$. By Remark 1 , if $n=11$ and $\alpha(H)=4$, then $H=Q$.

Lemma 8 Let $H$ be a triangle-free subcubic graph without isolated vertices that does not contain $Q$. Then $\beta^{\prime}(H) \leq \frac{3}{2} \alpha(H)$. Moreover, if equality holds then $H$ is a disjoint union of $C_{5}$ 's.

Proof. Since $\beta^{\prime}(H)=n-\alpha^{\prime}(H)$ where $n=|V(H)|$, it suffices to prove that $n-\alpha^{\prime}(H) \leq \frac{3}{2} \alpha(H)$. Now if $H$ has a perfect matching then $\alpha^{\prime}(H)=n / 2$ and the result follows immediately from Theorem 6 . Thus we may assume that $H$ has no 
perfect matching. Let $S$ be a Tutte set, that is, a subset of $V(H)$ such that $H-S$ has $c=|S|+n-2 \alpha^{\prime}(H)$ odd components. If we let $S$ be a set of maximal size with respect to this property then every component of $H-S$ is odd and hypomatchable. Let $H_{i}, 1 \leq i \leq c$ denote the components of $H-S$, and set $n_{i}=\left|V\left(H_{i}\right)\right|$.

Now $\alpha(H) \geq \sum_{i=1}^{c} \alpha\left(H_{i}\right) \geq \sum_{i=1}^{c}\left(n_{i}+1\right) / 3$ (using Lemma 7 ), and so we obtain

$$
\alpha(H) \geq(n-|S|+c) / 3=\left(2 n-2 \alpha^{\prime}(H)\right) / 3 .
$$

This implies the first assertion of the lemma. To show the second assertion, assume equality holds. Since $H$ contains no copy of $Q$ we know that $\sum_{i=1}^{c} \alpha\left(H_{i}\right)=$ $\sum_{i=1}^{c}\left(n_{i}+1\right) / 3$ implies that every component of $H-S$ is a 5-cycle. If $S=\emptyset$, then $H$ is the disjoint union of $c$-cycles, as claimed. Suppose that $x \in S$. Since $H$ is triangle-free, $x$ has at most two neighbors in each of the 5 -cycles, $H_{1}, \ldots, H_{c}$. So, in each $H_{i}$ we can choose two non-neighbors $y_{i}, y_{i}^{\prime}$ of $x$ such that $y_{i} y_{i}^{\prime} \notin E(H)$. Since the set $\{x\} \cup \bigcup_{i=1}^{c}\left\{y_{i}, y_{i}^{\prime}\right\}$ is independent, we have $\alpha(H) \geq 1+2 c>\sum_{i=1}^{c}\left(n_{i}+1\right) / 3$, a contradiction to our assumption of equality. This completes the proof.

Proof of Theorem 2. By Claim 4 we know that $T(G)$ is triangle-free, and we may assume that $T(G)$ has no isolated vertices. We claim that $T(G)$ does not contain $Q$, in which case the proof is complete by Lemma 8 and Claim 5 . Suppose on the contrary that $T(G)$ does contain $Q$. Observe that $Q$ contains a 5-cycle $C$ (marked in bold in Fig. 2) together with another vertex $x$ that is adjacent to two non-adjacent vertices of $C$ (see Fig. 2). By Claim 5 we know that $C$ corresponds to a 5 -wheel $W$ in $G$. Then since $G$ is flat, it is not possible for another triangle $x$ to share an edge with two non-adjacent triangles in $W$, as each must be adjacent to $x$ along a rim edge. Thus $T(G)$ cannot contain $Q$, as required.

\section{$3 \quad$ Planar graphs}

We are now ready to prove Theorem 1 . Let $G$ be a counter-example with the smallest $|V(G)|+|E(G)|$. For graphs with at most 4 vertices the statement is evident, so $|V(G)| \geq 5$. Also by the minimality we have:

(S1) Each edge of $G$ belongs to a triangle;

(S2) For each triangle B, at least 2 edges of $B$ belong to other triangles.

By Theorem 2, $G$ is not flat. This means that there is a separating triangle $B_{0}$ such that some other triangles are inside $B_{0}$ and some outside. We choose $B_{0}$ to be a separating triangle with minimum interior, so that in particular the subgraph of $G$ induced by the vertices inside and on $B_{0}$ is a flat plane graph without separating triangles (and $B_{0}$ is one of its triangles). We denote this subgraph by $G_{1}$ and the 
graph $G_{1}-E\left(B_{0}\right)$ by $G_{1}^{\prime}$. Similarly the "outside" subgraph $G-G_{1}^{\prime}$ will be denoted by $G_{2}$, and $G-G_{1}=G_{2}-E\left(B_{0}\right)$ will be denoted by $G_{2}^{\prime}$.

For $i=1,2$, let $\nu_{i}=\nu\left(G_{i}^{\prime}\right)$ and $\tau_{i}=\tau\left(G_{i}^{\prime}\right)$. We now derive some properties of $G$.

(S3) Let $W$ be a 5-wheel in $G$ and $(x, y, z)$ be a 3-face of $W$. Then it cannot happen that all vertices of $G$ not in $W$ are inside $(x, y, z)$ while all of $W-\{x, y, z\}$ are outside $(x, y, z)$.

Proof. Let $x$ be the vertex of degree 5 in $W$ and let the rim of $W$ be $(y, u, v, w, z)$. Suppose that only the face $(x, y, z)$ contains vertices of $G-W$. Let $G^{\prime}:=G-u-v-w$. Then $\nu\left(G^{\prime}-x y\right) \leq \nu(G)-2$, since any set of edge-disjoint triangles in $G-x y$ can be complemented by $(x, y, u)$ and $(x, v, w)$. So, if $T\left(G^{\prime}-x y\right)$ is not a disjoint union of $C_{5}$ s, then by the minimality of $G, \tau\left(G^{\prime}-x y\right)<3(\nu(G)-2) / 2$, and we can add 3 edges including $x y$ (e.g. $\{x y, x v, x w\}$ ) that cover all remaining triangles of $G$. Thus, $T\left(G^{\prime}-x y\right)$ is the disjoint union of $C_{5}$ s. If one of the corresponding 5 -wheels in $G$ contains the edge $x z$, then we can choose a covering of this wheel with 3 edges using $x z$ and cover the triangles of $W$ not containing $x z$ with two edges, one of which is $x y$. So, $x z$ does not belong to triangles in $G^{\prime}-x y$. By symmetry, we find that $T\left(G^{\prime}-x z\right)$ is the disjoint union of $C_{5}$ s and $x y$ does not belong to triangles in $G^{\prime}-x z$. Therefore $T\left(G^{\prime}-x y\right)=T\left(G^{\prime}-x z\right)=T\left(G^{\prime}-x y-x z\right)$ is a disjoint union of $C_{5}$ s. Now a similar argument shows that $y z$ does not belong to triangles in $G^{\prime}-x y-x z$. It follows that $T=T(G)$ is a disjoint union of $C_{5}$ s, a contradiction.

Everywhere below we assume that $B_{0}=(x, y, z)$.

(S4) $\nu(G)=\nu_{1}+\nu_{2}+1$.

Proof. Indeed, since the edges in $B_{0}$ do not participate in triangles of $G_{1}^{\prime}$ and $G_{2}^{\prime}$, $\nu(G) \geq \nu_{1}+\nu_{2}+1$. On the other hand, suppose $\nu(G) \geq \nu_{1}+\nu_{2}+2$. By definition, $\tau(G) \leq \tau_{1}+\tau_{2}+\left|E\left(B_{0}\right)\right|=\tau_{1}+\tau_{2}+3$. By the minimality of $G, \tau_{1}+\tau_{2} \leq \frac{3}{2}\left(\nu_{1}+\nu_{2}\right)$ with equality only if all components of $T\left(G-E\left(B_{0}\right)\right)$ are $C_{5}$. So, we may assume that all components of $T\left(G-E\left(B_{0}\right)\right)$ are $C_{5}$ and that $\nu(G)=\nu_{1}+\nu_{2}+2$, since otherwise we are done. By $(\mathrm{S} 1)$ and $(\mathrm{S} 2), \nu_{1} \geq 1$ and $\nu_{2} \geq 1$, and hence each of $G_{1}^{\prime}$ and $G_{2}^{\prime}$ contains at least one 5-wheel. Moreover, each such 5-wheel shares an edge with a triangle containing an edge of $B_{0}$, since otherwise, we can delete the edges of a 5 -wheel $W$ and use induction for $G-E(W)$.

Case 1: Every 5-wheel in $G_{1}^{\prime}$ shares at most one edge with triangles containing edges of $B_{0}$. Then we can cover all triangles in $G_{1}$ apart from $B_{0}$ with $3 k$ edges, where $k$ is the number of (edge disjoint) 5 -wheels in $G_{1}^{\prime}$, which is equal to $\nu_{1} / 2$. On the other hand, $\nu\left(G_{2}\right) \leq \nu-\nu_{1}$ and by the minimality of $G, \tau\left(G_{2}\right)<1.5\left(\nu-\nu_{1}\right)$, unless $T\left(G_{2}\right)$ is a union of disjoint $C_{5}$. But $G_{2}$ is obtained from $G_{2}^{\prime}$ by adding the edges of $B_{0}$, and the edges of a triangle in a 5 -wheel (or any two edges) do not cover all triangles in this wheel. So if $T\left(G_{2}^{\prime}\right)$ is the union of disjoint $C_{5}$, then $T\left(G_{2}\right)$ is not. This finishes the case.

Case 2: Some 5-wheel $W$ in $G_{1}^{\prime}$ shares more that one edge with triangles containing edges of $B_{0}$. By the choice of $B_{0}$, the outside face of $W$ is its 5-face. If a triangle 
$D$ in $G$ shares an edge with $W$ and shares an edge with $B_{0}$, then $W$ shares a vertex with $B_{0}$. Furthermore, $W$ cannot share more than one vertex with $B_{0}=(x, y, z)$, since otherwise $G[W]$ contains an edge not in $W$ and adding any edge to $W$ creates a $K_{4}$. We may assume that $x$ belongs to $W$ and $y$ and $z$ not. Then there is no triangle containing $y z$ sharing an edge with $W$. Let the rim of $W$ be $\left(x, x_{1}, x_{2}, x_{3}, x_{4}\right)$ and the center be $x_{0}$. Since the center of $W$ is inside its rim (we call such a 5 -wheel normal) and $G_{1}$ is flat, we may assume that the triangles containing an edge of $W$ and an edge of $B_{0}$ are $\left(x, y, x_{1}\right)$ and $\left(x, z, x_{4}\right)$. The edges $y x_{1}$ and $z x_{4}$ cannot belong to the same 5 -wheel in $G_{1}^{\prime}$, since it would be normal and $y x_{1}$ and $z x_{4}$ would be rim edges, so together with $y z$ we would find a $K_{4}$ in $G$. Hence, there is a set $F$ of $\nu_{1}+2$ edgedisjoint triangles in $G_{1}-z y$ consisting of triangles $\left(x, y, x_{1}\right),\left(x, z, x_{4}\right),\left(x_{0}, x_{1}, x_{2}\right)$, $\left(x_{0}, x_{3}, x_{4}\right)$, and two triangles from each other 5 -wheel in $G_{1}-z y$ (see Figure 3$)$. It follows that $\nu\left(G_{2}-x y-x z\right) \leq \nu_{2}$. Now $T\left(G_{2}^{\prime}\right)$ is a disjoint union of $C_{5}$, and $y$ and $z$ do not both belong to the same 5 -wheel in $G_{2}^{\prime}$ otherwise they form a $K_{4}$. Thus if $y z$ is in a triangle in $G_{2}-x y-x z$ then we could find $\nu_{2}$ triangles in $G_{2}^{\prime}$ avoiding it, giving a total of $|F|+\nu_{2}+1=\nu_{1}+\nu_{2}+3$ edge-disjoint triangles, a contradiction. Therefore $y z$ does not belong to a triangle in $G_{2}$ other than $B_{0}$. There is at most one triangle, say $(y, z, u)$ in $G_{1}$ distinct from $B_{0}$ that contains $z y$. If there is no such triangle, or $(y, z, u)$ shares an edge with a 5 -wheel in $G_{1}^{\prime}$, then we find a covering containing $1.5 \nu_{2}$ edges in $G_{2}^{\prime}$, edges $x y, x z$ and $1.5 \nu_{1}$ edges in $G_{1}^{\prime}$ covering all triangles in 5-wheels of $G_{1}^{\prime}$ and the triangle $(y, z, u)$, if it exists. See Figure 3, where the bold edges are an example of part of a suitable cover. But if $(y, z, u)$ exists and does not share any edge with a 5 -wheel in $G_{1}^{\prime}$, then $\nu\left(G_{1}\right) \geq \nu_{1}+3$, a contradiction.

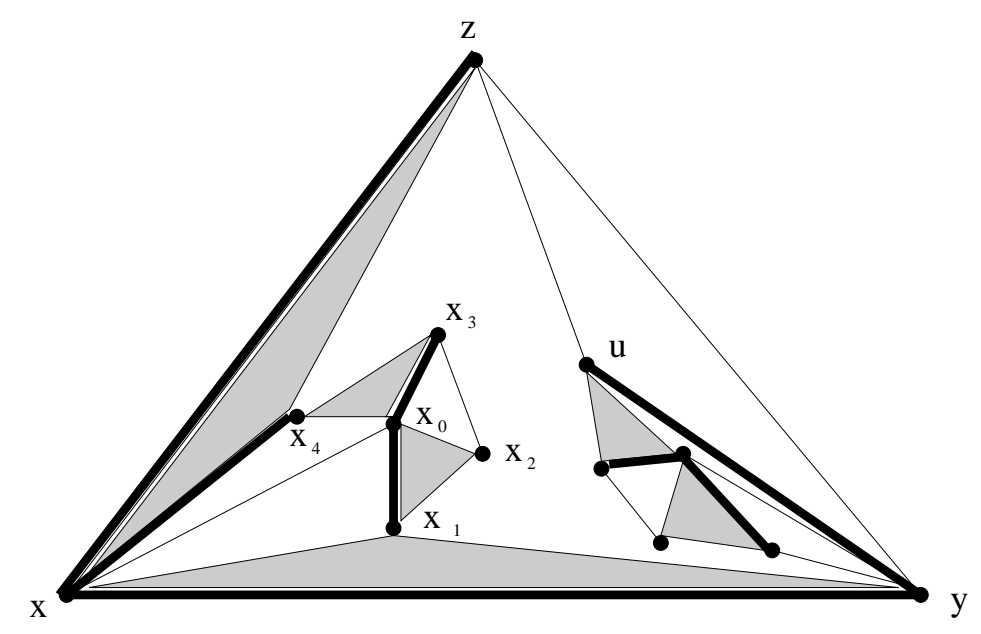

Figure 3: The shaded triangles form an example of the set $F$. 
Property (S4) implies also

(S5) For $i=1,2, \nu\left(G_{i}\right)=\nu_{i}+1$.

(S6) For every $X \subset E\left(B_{0}\right), \nu\left(G_{1}-X\right)+\nu\left(G_{2}-\left(E\left(B_{0}\right)-X\right)\right) \leq \nu_{1}+\nu_{2}+1$.

(S7) For every edge e of $B_{0}$, either $\nu\left(G_{1}^{\prime}+e\right)=\nu_{1}+1$ or $\nu\left(G_{2}^{\prime}+e\right)=\nu_{2}+1$.

Proof. Assume that $\nu\left(G_{1}^{\prime}+x y\right)=\nu_{1}$ and $\nu\left(G_{2}^{\prime}+x y\right)=\nu_{2}$. If neither of $T\left(G_{1}^{\prime}+x y\right)$ and $T\left(G_{2}^{\prime}+x y\right)$ is a disjoint union of $C_{5}$ s, then we can get a triangle cover for $G$ by adding edges $y z$ and $x z$ to the union of optimum triangle coverings in $G_{1}^{\prime}+x y$ and $G_{2}^{\prime}+x y$. This would imply that

$$
\tau(G) \leq 2+\left(1.5 \nu_{1}-0.5\right)+\left(1.5 \nu_{2}-0.5\right)=1.5\left(\nu_{1}+\nu_{2}\right)+1=1.5 \nu(G)-0.5 .
$$

So, there is $j \in\{1,2\}$ such that $T\left(G_{j}^{\prime}+x y\right)$ is a disjoint union of $C_{5}$ s.

Case 1: Neither of $x z$ and $y z$ belongs to a triangle in $G_{j}$ other than $B_{0}$. If $x y$ also does not belong to a triangle in $G_{j}$ other than $B_{0}$, then each triangle of $G$ belongs either to $G_{j}^{\prime}$ or to $G_{3-j}$ which are edge-disjoint. So we are done by the minimality of $G$. So, $x y$ belongs to a 5 -wheel in $G_{j}^{\prime}+x y$, and hence there is a set $S$ of $1.5 \nu_{j}$ edges covering all triangles in $G_{j}^{\prime}+x y$ such that $x y \in S$. If $T\left(G_{3-j}\right)$ is not a disjoint union of $C_{5} \mathrm{~s}$, then there is $S^{\prime} \subset E\left(G_{3-j}\right)$ covering all triangles with $\left|S^{\prime}\right|<1.5\left(\nu_{3-j}+1\right)$; so that $\left|S \cup S^{\prime}\right|<1.5 \nu(G)$. Suppose that $T\left(G_{3-j}\right)$ is a disjoint union of $C_{5}$ s. Since $B_{0}$ is a triangle in $G_{3-j}$, and $x y \in S$, we again need fewer than $1.5 \nu_{3-j}$ edges to cover triangles in $G_{3-j}-x y$.

Case 2: The edge $x z$ belongs to a triangle in $G_{j}$ other than $B_{0}$ (say, $(x, z, u)$ ), and the edge $y z$ does not. We claim that

$$
\nu\left(G_{j}^{\prime}+x z\right) \geq \nu_{j}+1 .
$$

If (1) does not hold, then some 5-wheel $W$ in $G_{j}^{\prime}+x y$ does not have two edge-disjoint triangles that are also disjoint from $(x, z, u)$ and do not contain edge $x y$. Since $x z \notin E\left(G_{j}^{\prime}+x y\right), W$ has at most two common vertices with $(x, z, u)$, otherwise $G$ contains a $K_{4}$. Thus $x y$ is an edge of $W$ and some edge $e \in\{x u, z u\}$ is an edge of $W$. If $e=z u$, then $W$ contains all of $(x, z, u)$. So, $e=x u$. Since $W-x y-x u$ has no two edge-disjoint triangles, $x$ is the center of $W$. Since $G[\{x, y, z, u\}] \neq K_{4}, u y \notin E(G)$. Then there is no face of $W$ to put $z$ so that it is adjacent to all of $x, y$ and $u$. So, (1) holds.

By (1) and (S6), $\nu\left(G_{3-j}-x z\right)=\nu_{3-j}$. In our case (i.e. $y z$ is not in a triangle in $G_{j}$ other than $\left.B_{0}\right)$ we also have $\nu\left(G_{j}-x z\right)=\nu_{j}$. So we can get a triangle cover for $G$ by adding edge $x z$ to the union of optimum triangle coverings in $G_{1}-x z$ and $G_{2}-x z$. This yields $\tau(G) \leq 1.5 \nu_{1}+1.5 \nu_{2}+1<1.5 \nu(G)$.

Case 3: Edge $x z$ belongs to a triangle $(x, z, u)$ in $G_{j}$ other than $B_{0}$ and the edge $y z$ belongs to a triangle $(y, z, v)$ in $G_{j}$ other than $B_{0}$. Since $G$ is $K_{4}$-free, $v \neq u$. Similarly to Case 2, we claim that

$$
\nu\left(G_{j}\right) \geq \nu_{j}+2,
$$


which would contradict (S4). If (2) does not hold, then some 5 -wheel $W$ in $G_{j}^{\prime}+x y$ does not have two edge-disjoint triangles that are also disjoint from $(x, z, u)$ and $(y, z, v)$. Then $W$ contains an edge $e_{1} \in\{x u, u z\}$ and an edge $e_{2} \in\{y v, v z\}$, and at least one of them is incident to the center of $W$. If $e_{1}=x u$ then $z \notin W$, otherwise $W+z x$ contains $K_{4}$. Thus $e_{2}=y v$ and one of $\{x, u, v, y\}$ is the center of $W$, but then we find a $K_{4}$ containing $z$. This contradiction shows that $e_{1}=u z$, and similarly $e_{2}=v z$. Then $z$ is the center of $W$ and $x, y \notin W$. Since $x y \in E(G), x$ and $y$ are in the same face of $W$. Since the set $\{x, y\}$ is adjacent to $z, u$, and $v$, this face should be $(u, z, v)$. In particular, the triangle $(u, z, v)$ separates $(x, z, y)$ from all other vertices of $W$. So, by the choice of $(x, z, y), j=2$. If a face $F$ of $W$ distinct from $(u, z, v)$ contains a vertex not in $W$, then by (S1) it contains a triangle distinct from itself. Since $T\left(G_{j}^{\prime}+x y\right)$ is a disjoint union of $C_{5} \mathrm{~s}, F$ contains a 5 -wheel $W^{\prime}$ distinct from $W$ and hence edge-disjoint from $W$. So, deleting the edges of $W^{\prime}$ from $G$ does not destroy other triangles of $G$, and we can apply induction hypothesis to $G-E\left(W^{\prime}\right)$, a contradiction. Thus no face of $W$ apart from $(u, z, v)$ contains vertices. Now we are done by (S3).

Final calculations By $(\mathrm{S} 7)$, there is $j \in\{1,2\}$ such that $\nu\left(G_{j}^{\prime}+x y\right)=\nu_{j}+1$. Then by $(\mathrm{S} 6), \nu\left(G_{3-j}-x y\right)=\nu_{3-j}$. Then again by $(\mathrm{S} 7), \nu\left(G_{j}^{\prime}+x z\right)=\nu_{j}+1$ and $\nu\left(G_{j}^{\prime}+z y\right)=\nu_{j}+1$, and again by $(\mathrm{S} 6), \nu\left(G_{3-j}-x z\right)=\nu_{3-j}$ and $\nu\left(G_{3-j}-z y\right)=$ $\nu_{3-j}$. By the minimality of $G$ there is an edge-cover $S_{j}$ of triangles in $G_{j}$ with $\left|S_{j}\right| \leq 1.5\left(1+\nu_{j}\right)$ (with equality only if $T\left(G_{j}\right)$ is the disjoint union of $C_{5} \mathrm{~s}$ ). Since $S_{j}$

covers $B_{0}$, we may assume that $x y \in S_{j}$. By above, there is an edge-cover $S_{3-j}$ of triangles in $G_{3-j}-x y$ with $\left|S_{3-j}\right| \leq 1.5 \nu_{3-j}$ (with equality only if $T\left(G_{3-j}-x y\right.$ ) is the disjoint union of $\left.C_{5} \mathrm{~s}\right)$. So, if at least one of $T\left(G_{j}\right)$ and $T\left(G_{3-j}-x y\right)$ is not the disjoint union of $C_{5} \mathrm{~s}$, then we are done. Suppose now that they both are. If the 5 -wheels of $G_{3-j}-x y$ contain neither $x z$ nor $y z$, then $T(G)$ is the disjoint union of $C_{5}$ s, and we are done. So, we may assume that $x z$ is in some 5 -wheel in $G_{3-j}-x y$. Note that there is an edge-cover $S_{j}^{\prime}$ of triangles in $G_{j}$ with $\left|S_{j}^{\prime}\right|=1.5\left(1+\nu_{j}\right)$ containing $x z$. Furthermore, if we delete from $G_{3-j}-x y$ the edge $x z$, we destroy one of the 5 -wheels, and when we then add to it the edge $x y$, the resulting graph $G_{3-j}-x z$ is not the edge-disjoint union of 5 -wheels. Since $\nu\left(G_{3-j}-x z\right)=\nu_{3-j}$, we are done.

\section{Appendix}

Here for completeness we show that if $G$ is a triangle-free subcubic graph on 11 vertices with independence number 4 , then it is isomorphic to the graph $Q$ shown in Figure 2.

Since $G$ has 11 vertices we know that it has a vertex $a$ with even degree. If $d(a)=0$ then $G-a$ is a graph with 10 vertices, that contains no triangle and no independent set of size 4 , contradicting the fact that the Ramsey number $R(3,4)$ is 9 . Therefore $d(a)=2$. Let $b$ and $c$ denote the neighbours of $a$. Then $G^{\prime}=G-\{a, b, c\}$ 
has 8 vertices, no triangle and no independent set of size 4 . Thus $G^{\prime}$ is an extremal Ramsey graph for $R(3,4)$, which means that it shows $R(3,4) \geq 9$. A complete list of such graphs is known (see e.g. [9]). They are: the graph $H$ obtained by removing the vertex $u$ of degree 2 in $Q$ together with its neighbours $v$ and $w$ (see Figure 2), the graph $H_{1}=H+p q$, and $H_{2}=H_{1}+r s$.

Observe that in $H_{2}$ (and therefore also in $H$ and $H_{1}$ ), for each $y \in\{p, q, r, s\}$ there exists an independent set $S_{y}$ of size 3 that contains $y$ and no element of $\{p, q, r, s\} \backslash\{y\}$. Since there must be an edge of $G$ from $\{b, c\}$ to $S_{y}$ (otherwise $G$ contains an independent set of size 5), and the two vertices in $S_{y} \backslash\{y\}$ each have degree 3 in $H$, the only possibility is that $G^{\prime}=H$ and each of $b$ and $c$ has two neighbours in $\{p, q, r, s\}$. Since $p s$ and $q r$ are edges and $G$ is triangle-free, we conclude that $G$ is isomorphic to $Q$.

\section{References}

[1] S. Aparna Lakshmanan, Cs. Bujtás, Zs. Tuza, Small edge sets meeting all triangles of a graph, submitted.

[2] G. Chapuy, M. DeVos, J. McDonald, B. Mohar, D. Scheide, Packing triangles in weighted graphs, submitted.

[3] Q. Cui, P. E. Haxell and W. Ma, Packing and covering triangles in planar graphs, Graphs Combin. 25 (2009) 817-824.

[4] S. Fajtlowicz, On the size of independent sets in graphs, Congr. Numer. 21 (1978) 269-274.

[5] P. Haxell, Packing and covering triangles in graphs, Discrete Math. 195 (1999) $251-254$.

[6] P. Haxell and Y. Kohayakawa, Packing and covering triangles in tripartite graphs, Graphs Combin. 14 (1998) 1-10.

[7] P. Haxell, A. Kostochka and S. Thomassé, A stability theorem on fractional covering of triangles by edges, European J. Comb. to appear

[8] M. Krivelevich, On a conjecture of Tuza about packing and covering of triangles, Discrete Math. 142 (1995) 281-286.

[9] B. McKay, Combinatorial data, http://cs.anu.edu.au/people/bdm/data/

[10] W. Stanton, Some Ramsey-type numbers and the independence ratio, Trans. Amer. Math. Soc. 256 (1979) 353-370.

[11] Zs. Tuza, Conjecture, Finite and Infinite Sets, Eger, Hungary 1981, A. Hajnal, L. Lovász, V. T. Sós (Eds.), Proc. Colloq. Math. Soc. J. Bolyai, Vol. 37, NorthHolland, Amsterdam, (1984), 888.

[12] Zs. Tuza, A conjecture on triangles of graphs, Graphs Combin. 6 (1990) 373-380. 